\title{
POLÍTICAS PÚBLICAS E PREVENÇÃO DO SUICÍDIO NO BRASIL
}

Victor Mauro Gonçalves Setti

Só existe um problema filosófico realmente sério: o suicídio. Julgar se a vida vale ou não vale a pena ser vivida é responder à pergunta fundamental da filosofia.

- A. Camus, O Mito de Sísifo

\begin{abstract}
RESUMO
O presente trabalho procura entender a razão pela qual as políticas públicas de prevenção do suicídio não recebem a atenção necessária do estado brasileiro, levantando os desafios representados pelo tabu ao redor do tema, abordagem inadequada pela mídia, má capacitação dada aos profissionais de saúde básica e subnotificação dos casos. A partir de uma revisão da literatura publicada sobre o assunto, procura-se identificar os tipos de prevenção do suicídio e suas diferentes abordagens ao longo do tempo, apontar suas limitações e levantar a necessidade de um plano nacional de prevenção do suicídio no Brasil, reconhecendo os desafios abarcados pela questão.
\end{abstract}

Palavras-chave: suicídio, políticas públicas, prevenção, estratégia nacional.

\begin{abstract}
The following study tries to understand the reason behind the absence of public policies focusing in suicide prevention in Brazil, bringing up the challenges represented by the taboo around the topic, the inadequate approach given by the media, the inefficient training of nonspecialized health workers and the underreporting of the events. Based on reviewing the published literature, it seeks to identify types of suicide prevention and its different approaches over time, pointing out its limitations and arguing in favor of a National Suicide Prevention Strategy for Brazil, identifying the challenges brought by the theme.
\end{abstract}

Keywords: suicide, public policies, prevention, national strategy. 


\section{Introdução}

Cerca de 800 mil pessoas se suicidam por ano em todo o mundo atualmente, uma taxa de 11,4 mortes para cada 100 mil habitantes, segundo dados da Organização Mundial da Saúde (OMS $)^{1}$. Outras milhões de pessoas experienciam comportamentos suicida todo ano, e para cada indivíduo que comete o suicídio, ficam para trás muitos outros "cujas vidas resultam profundamente afetadas desde o ponto de vista emocional, social e econômico" (DE LEO; BERTOLOTE; LESTER, 2003, p. 201). De acordo com a agência das Nações Unidas, em 2012, 75\% dos casos ocorreram em países onde a renda é considerada baixa ou média. Além disso, entre jovens adultos nas idades de 15 a 29 anos, o suicídio representa 8,5\% de todas as mortes e é a segunda maior causa nesse grupo, perdendo apenas para acidentes de trânsito (WHO, 2016, p. 36).

As taxas de suicídio no Brasil podem ser consideradas baixas quando comparadas às médias mundiais, com uma taxa global de 5,5 suicídios por 100 mil habitantes, em 2015 (BRASIL, 2017). Entretanto, ao se analisar o número de casos de suicídio por ano, o Brasil se encontra entre os dez países do mundo onde há mais mortes por suicídio. Entre 2005 e 2015, o total de suicídios no Brasil passou de 8.550 para 11.178 , um aumento de $31 \%$, superior ao crescimento da população nesse mesmo período, de $11 \%$.

Pelo fato de ser um país de proporções continentais, o Brasil enfrenta uma situação ainda mais complexa ao se tratar do tema, considerando sua extensão territorial e diversidade cultural. Koch \& Oliveira lembram que o suicídio é ainda mais forte em grupos marginalizados e discriminados da sociedade, como gays, indígenas e imigrantes $(\mathrm{KOCH} \&$ OLIVEIRA, 2015, p. 161-172) e em certas cidades e regiões, bem como em alguns grupos populacionais, como, por exemplo, jovens em grandes cidades, indígenas do Centro-Oeste e do Norte e entre lavradores do interior do Rio Grande do Sul - onde as taxas se aproximam e até superam a de países do leste europeu e da Escandinávia, como lembra Botega $(2007$, p. 7). Somado a isso, está o fato de haver uma subnotificação de eventos, e estima-se que o número de tentativas de suicídio supere em pelo menos 10 vezes o de suicídios.

Apesar dos dados, as políticas públicas de prevenção do suicídio ainda são muito pouco abordadas no Brasil, e raramente são objetivo de ações efetivas do Estado, mesmo que as taxas anuais sigam crescendo. Machado, Leite e Bando (2014, p.337) lembram que entre os entraves desta questão estão o tabu em torno do tema, o atendimento negligenciado, a

\footnotetext{
${ }^{1}$ Informação disponível em http://www.who.int/mental_health/prevention/suicide/suicideprevent/en/. Acesso em 30 de março de 2017.
} 
abordagem sensacionalista da mídia, o acesso aos métodos para cometer suicídio e o abuso de substâncias químicas. O objetivo desse texto é comparar as abordagens dadas à prevenção do suicídio, considerando o contexto internacional e, principalmente, nacional. A partir do levantamento bibliográfico de autores como José Manoel Bertolote e Neury José Botega, procura-se compreender a importância de uma estratégia nacional de prevenção do suicídio. É realizada uma abordagem qualitativa, visando o aprofundamento da compreensão do comportamento suicida e sua prevenção, levantando fatores causais e possíveis soluções. A pesquisa se dá de forma exploratória, com o objetivo de proporcionar maior familiaridade com o tema do suicídio, tornando-o mais explícito.

\section{Políticas Públicas, Suicídio e Prevenção}

Compreende-se como políticas públicas as atividades governamentais que exercem alguma influência sobre a vida dos cidadãos (PETERS, 2013, p. 4). Na definição de Thomas D. Dye (apud SOUZA, 2006, p. 24), política pública é "o que o governo escolhe fazer ou não fazer", ressaltando-se que a omissão do Estado também é uma decisão com impacto na vida dos cidadãos.

A definição de suicídio proposta pela OMS (BERTOLOTE, 2012, p. 21 apud, OMS,1998) o considera como o ato deliberado de pôr fim à própria vida. O suicídio é um processo que se inicia com a "ideação suicida", ou seja, considerações vagas sobre a morte e sobre morrer, que dependendo da intensidade, podem evoluir para um "plano suicida" e resultar num ato suicida, que pode ou não ter um desfecho fatal.

Considera-se que o suicídio possua uma condição de multicausalidade, sendo um comportamento multifatorial e multideterminado; no entanto, Bertolote acredita que um conhecimento aprofundado dos fatores causais dos comportamentos suicidas seja de grande relevância para a sua prevenção (BERTOLOTE, 2012, p. 68). O autor diz que é importante, tanto do ponto de vista conceitual quanto prático, diferenciar fatores predisponentes de fatores precipitantes. O primeiro se refere à determinadas constituições genéticas, biológicas e individuais, enquanto o segundo está ligado a fatores ambientais, como fácil acesso a métodos de suicídio, e situações estressantes, perdas significativas, luto, dentre outros.

Por outro lado, entende-se por prevenção qualquer medida que vise interceptar a causa de uma doença antes que ela atinja um indivíduo. Bertolote (2012, p. 88) assinala que Mrazek e Haggerty propuseram três níveis de prevenção: Universal, Seletiva e Indicada. A primeira, destinada a toda a população, visa impedir o início de um dado comportamento; um 
exemplo de prevenção universal do suicídio é o Dia de Prevenção do Suicídio, onde toda a população é informada sobre a questão. A segunda é destinada a populações ou indivíduos que ainda não apresentaram o comportamento-alvo, visando impedir que este se instale. Um exemplo de prevenção seletiva de comportamentos suicidas é a busca ativa de indivíduos com certos transtornos mentais associados à questão. Por último, o terceiro diz respeito a populações ou indivíduos com risco considerável que já manifestaram o comportamento. Um exemplo de prevenção indicada de comportamento suicida é o acompanhamento de indivíduos que já tentaram o suicídio (BERTOLOTE, 2012, p. 88-89).

Especificamente sobre a prevenção do suicídio, os primeiros esforços datam do início do século XX. No entanto, eram inspirados por princípios filantrópicos, carecendo de base científica. Bertolote (2012, p. 93-96) aponta que Gunnel e Frankel fizeram um levantamento da literatura publicada entre 1975 e 1994, e assinalaram uma série de limitações na abordagem filantrópica, como a ausência de um objetivo e público-alvo claros, o pequeno número de sujeitos incluídos, o curto tempo de acompanhamento dos indivíduos e descrições superficiais da estratégia de intervenção. Ainda hoje pode-se observar programas de prevenção do suicídio inspirados nesses princípios filantrópicos. David Lester (MACHADO; LEITE; BANDO, 2014, p. 345) aborda os centros de prevenção nos EUA, com o atendimento via telefone de pessoas em crise, mas o serviço pode ser considerado estritamente passivo, sem uma busca ativa de pessoas com comportamentos suicidas.

\section{Estratégia Nacional de Prevenção do Suicídio}

Diferente da abordagem filantrópica, e com eficácia comprovada, estão os programas de prevenção de suicídio associados à uma estratégia nacional. O surgimento de programas de prevenção do suicídio na saúde pública data de uma reunião internacional de peritos, em 1994, em Banff, Canadá, convocada pelo Conselho para Assuntos Econômicos e Sociais da ONU, e pelo Departamento de Saúde Mental da OMS. Resultou na publicação, em 1996, de um documento intitulado Prevention of Suicide: Guidelines for the Formulation and Implementation of National Strategies. O documento teve a dupla importância de ser a primeira vez que organismos internacionais reconheciam os comportamentos suicidas como um importante problema social e econômico, com grande impacto na saúde pública, e por refletir uma abordagem sólida com base científica reconhecida em seus peritos (BERTOLOTE, 2012, p. 98). Em 2004, com o apoio da Suicide Prevention International da 
OMS, um grupo de peritos oriundos de 15 países se reuniram e confirmaram cinco pontos prioritários para o desenvolvimento de atividades preventivas do comportamento suicida:

- Restrição do acesso a métodos empregados em comportamentos suicidas;

- Abordagem adequada pelos meios de comunicação;

- Tratamento adequado de transtornos mentais;

- Programas adequados de educação e informação;

- Busca ativa e acompanhamento de indivíduos com comportamento suicida ${ }^{2}$.

Tem aumentado o número de países com estratégias nacionais de prevenção do suicídio, seja com estratégias amplas, localizadas ou descentralizadas. Botega (2006, p. 218) aponta que, de modo geral, esses países possuem programas educacionais para informar a população, buscam um melhor atendimento dos casos, incentivam pesquisas na área, oferecem treinamento para escolas, mídia e equipes de saúde, entre outros. Segundo o World Health Statistics de 2016, publicado pela OMS (WHO, 2016, p. 62), ocorreu uma redução global de $9 \%$ no número de mortes por suicídio e uma queda de $21 \%$ na taxa mundial, entre 2000 e 2012, apesar de um aumento das taxas em 50 países, quando tomados individualmente. A organização aponta quais estratégias nacionais de ação, entre outros fatores, contribuíram para as melhorias observadas.

\section{Brasil: As Diretrizes Nacionais}

No Brasil, até pouco tempo, o suicídio não era visto como um problema de saúde pública, e encontrava-se na sombra dos elevados índices de homicídio e de acidentes de trânsito, entre as causas externas da mortalidade. Botega (2006, p. 218) afirma que o Brasil deu os primeiros passos no sentido de lançar um plano nacional de prevenção do suicídio ao montar, em 2005, um grupo de trabalho para este fim, formado por representantes do governo, de entidades da sociedade civil e de universidades. Daí surgiram as Diretrizes Brasileiras para um Plano Nacional de Prevenção do Suicídio (BRASIL, 2006) e que orientam quais deveriam ser os objetivos para uma estratégia nacional:

1 Desenvolver estratégias de promoção de qualidade de vida, de educação, de proteção e de recuperação da saúde e de prevenção de danos;

2 Desenvolver estratégias de informação, de comunicação e de sensibilização da sociedade de que o suicídio é um problema de saúde pública que pode ser prevenido;

3 Organizar linha de cuidados integrais (promoção, prevenção, tratamento e recuperação) em todos os níveis de atenção, garantindo o acesso às diferentes modalidades terapêuticas;

\footnotetext{
${ }^{2}$ Mais recentemente, a OMS também sugere a introdução de políticas para a redução de danos causados pelo consumo de álcool.
} 
4 Identificar a prevalência dos determinantes e condicionantes do suicídio e tentativas, assim como os fatores protetores e o desenvolvimento de ações intersetoriais de responsabilidade pública, sem excluir a responsabilidade de toda a sociedade;

5 Fomentar e executar projetos estratégicos fundamentados em estudos de custoefetividade, eficácia e qualidade, bem como em processos de organização da rede de atenção e intervenções nos casos de tentativas de suicídio;

6 Contribuir para o desenvolvimento de métodos de coleta e análise de dados, permitindo a qualificação da gestão, a disseminação das informações e dos conhecimentos;

7 Promover intercâmbio entre o Sistema de Informações do SUS e outros sistemas de informações setoriais afins, implementando e aperfeiçoando permanentemente a produção de dados e garantindo a democratização das informações; e

8 Promover a educação permanente dos profissionais de saúde das unidades de atenção básica, inclusive do Programa Saúde da Família, dos serviços de saúde mental, das unidades de urgência e emergência, de acordo com os princípios da integralidade e da humanização.

O Brasil foi o primeiro país da América Latina a elaborar estratégias de prevenção do comportamento suicida (BOTEGA, 2007, p. 8), mas apesar de tal avanço, o país jamais entregou um Plano Nacional de Prevenção do Suicídio propriamente dito, enquanto as taxas de suicídio seguem crescendo anualmente, principalmente entre jovens (WAISELFISZ, 2014, p. 114).

\section{Desafios e Limitações do Caso Brasileiro}

É preciso reconhecer que existe um tabu em torno do suicídio, fato que impede os indivíduos de procurarem informação e ajuda, especialmente entre grupos marginalizados e descriminados. Existe também um tabu na mídia, que teme divulgar debates relativos ao tema e causar ondas de suicídio por imitação ou indução (Ibid., p. 113), e acaba omitindo informações, enquanto os tabloides tratam do assunto de maneira sensacionalista. Desmistificar o tema abre possibilidades de falar sobre o assunto de modo adequado, e reduz a subnotificação.

Outro fator importante é a capacitação de profissionais de saúde da atenção básica. Botega, Marín-león, Oliveira, Barros, Silva e Dalgalarrondo (2009, p. 2637), analisando o município de Campinas, em São Paulo, constataram que aproximadamente um quinto da população já havia apresentado ideação suicida ao longo da vida, mas que, de cada três casos de tentativas de suicídio, apenas um foi atendido pelo serviço médico. Carlos Estellita-Lins (apud MACHADO; LEITE; BANDO, 2014, p. 384) aponta que os serviços de emergência no Rio de Janeiro são marcados pela negligência na abordagem sobre suicídio, bem como a ausência de um treinamento dos profissionais. 
Por último, a produção acadêmica do assunto não acompanhou de forma proporcional o crescimento do número de casos, prejudicando a identificação de problemas e o levantamento de melhores soluções.

\section{Considerações Finais}

O Brasil carece de um Plano Nacional de Prevenção do Suicídio, definindo objetivos claros, embasado em evidências científicas e superando a falta de atendimento, tratamento e apoio adequados, e a deficiência nos métodos de identificação dos casos, que expõem a necessidade de políticas públicas eficientes na área da saúde pública para prevenção.

As Diretrizes lançam a base para o que deve vir a ser um Plano Nacional, no entanto, ainda não se encontram suficientemente implementadas e articuladas. Elas demonstram o aspecto multicausal do suicídio, incluindo pontos importantes, como a promoção de qualidade de vida e de educação, e, inclusive, procuram garantir a democratização das informações do Sistema Único de Saúde. É inegável a importância das Diretrizes, principalmente quando se considera que Brasil foi o primeiro país da América Latina a elaborar uma estratégia de prevenção do comportamento suicida, e, por isso, devem ser aperfeiçoadas, seguindo o exemplo de diversos países, onde estratégias nacionais bem conduzidas já possibilitaram significativas reduções dos casos de suicídio.

Fato interessante é que a OMS não se opõe à descriminalização do suicídio, atribuindo a esse fato a melhora no acesso de informação e certa superação do tabu em países que implementaram tal medida (WHO, 2016, p. 62). No entanto, embora exista uma propensão a se tratar o suicídio como uma questão de liberdade individual, é preciso ter consciência de que nem todos aqueles que dão fim a própria vida estão em condições emocionais e mentais de tomar decisões livres. Botega, Marín-león, Oliveira, Barros, Silva e Dalgalarrondo (2009, P. 219) lembra que pensar na prevenção do suicídio significa acreditar que é possível oferecer aos indivíduos outras possibilidades de enfrentamento das dificuldades ou patologias que os levam a buscar, nesse ato fatal, uma espécie de solução para seu sofrimento.

O suicídio deve ser reconhecido como um problema de saúde pública, mas que pode ser prevenido. É necessária uma resposta nacional, com políticas públicas multisetoriais que abranjam os setores da saúde, dentre outros, aperfeiçoando a identificação e acompanhamento de indivíduos com transtornos mentais, e restringindo o acesso a meios letais, prevenindo principalmente os casos impulsivos. As comunidades também têm um papel fundamental 
nesse trabalho de prevenção, oferecendo um suporte a indivíduos vulneráveis, em situações de crise, e contrabalanceando o peso das circunstâncias difíceis da vida. No entanto, o Estado não pode se omitir, e, no caso brasileiro, as bases já estão lançadas. 


\section{Referências Bibliográficas}

BERTOLOTE, José M. O suicídio e sua prevenção. São Paulo: Editora Unesp. 2012

BOTEGA, Neury J., MARÍN-LEÓN, Letícia., OLIVEIRA, Helenice B. de., BARROS, Marilisa B. de A., SILVA, Viviane F. da. \& DALGALARRONDO, Paulo. Prevalências de ideação, plano e tentativa de suicídio: um inquérito de base populacional em Campinas, São Paulo, Brasil. Cadernos de Saúde Pública, Rio de Janeiro, 25(12):2632-2638, 2009.

BOTEGA, Neury J., WERLANG, Blanca S. G., Cais, CARLOS F. da S. \& MACEDO, Mônica M. K. Prevenção do Comportamento Suicida. Revista Psico. Porto Alegre, V.37, n.3, p. 213-220, 2006.

BOTEGA, Neury José. Suicídio: saindo da sombra em direção a um Plano Nacional de Prevenção. Revista Brasileira de Psiquiatria, São Paulo,29(1), p. 7-8. 2007.

BRASIL. Ministério da Saúde. DATASUS. Informações de Saúde. Epidemiológicas e Morbidade. Disponível em: <www.datasus.gov.br/DATASUS/> Acesso em 12 de junho de 2017.

BRASIL. Ministério da Saúde. Portaria n 1.876 , de 14 de agosto de 2006. Institui Diretrizes Nacionais Para Prevenção do Suicídio, A Ser Implantadas em Todas As Unidades Federadas, Respeitadas As Competências das Três Esferas de Gestão. Brasília, 2006.

CAMUS, A. O Mito de Sísifo. 3ª ed. Rio de Janeiro: BestBolso, 2013.

DE LEO, D.; BERTOLOTE, J., \& LESTER, D. La violencia autoinfligida. In: E. G. Krug, L. L. Dahlberg, J. A. Mercy, A. B. Zuvi, \& R. Lozano. Informe mundial sobre la violencia y la salud. Washington: Organización Panamericama de la Salud, p. 201-231, 2003.

DISTRITO FEDERAL. Portaria $\mathrm{n}^{\circ}$ 185, de 12 de setembro de 2012. Institui As Normas Para Atenção às Emergências em Saúde Mental no âmbito do SUS-DF. Distrito Federal, DF, 13 set. 2012. n. 186.

FREY, K. Políticas públicas: um debate conceitual e reflexões referentes à prática da análise de políticas públicas no Brasil. Planejamento e políticas públicas, Rio de Janeiro, Instituto de Pesquisa Econômica Aplicada (IPEA), n. 21, p. 211-259, 2000.

KOCH, D. B.; OLIVEIRA, P. R. M. de . As políticas públicas para prevenção de suicídios. Revista Brasileira de Tecnologias Sociais , v. 2, p. 161-172, 2015.

MACHADO, M. F. S.; LEITE, C. K. S.; BANDO, D. H. Políticas Públicas de Prevenção do Suicídio no Brasil: uma revisão sistemática. Revista Gestão \& Políticas Públicas, São Paulo, vol. 4(2), p. 334-356, 2014 
PETERS, B. Guy. (1986) American Public Policy: promise and performance. California: CQ Press. 2013.

SOUZA, Celina. Políticas públicas: uma revisão da literatura. Sociologias, Porto Alegre, n. 16, p. 20-45, 2006

WAISELFISZ, J. J. Mapa da Violência 2014 - Os jovens do Brasil. Brasília, 2014. Disponível em http://www.mapa daviolencia.org.br/pdf2014/Mapa2014_JovensBrasil.pdf. Acesso em 30 de março de 2017.

WHO. World health statistics 2016: Monitoring health for the SDGs, sustainable development goals. France, 2016. 